The University of Southern Mississippi

The Aquila Digital Community

Faculty Publications

8-15-1990

\title{
Theory of Light Scattering by a Nonequilibrium Binary Mixture
}

J.C. Nieuwoudt

University of Southern Mississippi

B.M. Law

Kansas State University

Follow this and additional works at: https://aquila.usm.edu/fac_pubs

Part of the Physics Commons

\section{Recommended Citation}

Nieuwoudt, J., Law, B. (1990). Theory of Light Scattering by a Nonequilibrium Binary Mixture. Physical Review A, 42(4), 2003-2014.

Available at: https://aquila.usm.edu/fac_pubs/7448

This Article is brought to you for free and open access by The Aquila Digital Community. It has been accepted for inclusion in Faculty Publications by an authorized administrator of The Aquila Digital Community. For more information, please contact Joshua.Cromwell@usm.edu. 


\title{
Theory of light scattering by a nonequilibrium binary mixture
}

\author{
J. C. Nieuwoudt ${ }^{*}$ \\ Department of Physics and Astronomy, University of Southern Mississippi, Hattiesburg, Mississippi 39406 \\ B. M. Law \\ Department of Physics, Kansas State University, Manhattan, Kansas 66506 \\ (Received 4 January 1990)
}

\begin{abstract}
A binary mixture subject to a large temperature gradient is studied. Solutions of the evolution equations for the time-dependent fluctuation correlations are obtained using mode-coupling theory subject to the assumption that the mixture is not close to a critical point. From these results predictions for the light-scattering spectrum at the Rayleigh and Brillouin lines are made. The temperature gradient is shown to cause a large enhancement of the scattering amplitude at the Rayleigh line, whereas the effect at the Brillouin line is found to be small.
\end{abstract}

\section{INTRODUCTION}

Nonequilibrium phenomena in fluids have been the subject of much theoretical and experimental study. ${ }^{1}$ An aspect of these studies is the scattering of light by nonequilibrium fluids, which provides a sensitive technique to investigate nonequilibrium processes. Predictions for the light-scattering spectrum in one-component fluids in a steady-state subject to a large temperature gradient were made by Kirkpatrick et al. $^{2-5}$ using both modecoupling and kinetic theory of fluids. Similar predictions were made by Ronis and Procaccia ${ }^{6}$ using the theory of fluctuating hydrodynamics. Detailed studies were subsequently made by Schmitz and Cohen. ${ }^{7}$

The results of the predictions for one-component fluids can be summarized as follows: At the Rayleigh line the temperature gradient is predicted to enhance the equilibrium spectrum by an additional term proportional to $\left(\nabla T / q^{2}\right)^{2}$, where $\nabla T$ is the temperature gradient and $q$ is the wave number of the fluid fluctuations. Small gradients leave the Rayleigh line unaffected. Experimental investigations of the Rayleigh line confirming the theoretical predictions were performed by Law et al. ${ }^{8,9}$ The Brillouin lines are affected by both small and large temperature gradients. For small gradients the peak amplitudes predicted to become asymmetric by factors proportional to $(\widehat{\mathbf{q}} \cdot \nabla T) / q^{2}$. For large gradients the effects are more complicated. ${ }^{5}$ Experimental investigations of the Brillouin lines ${ }^{10,11}$ indicate agreement with the theoretical predictions but, due to complicating boundary effects, are less definitive.

In this paper we apply mode-coupling theory to study nonequilibrium effects in the light-scattering spectrum in a binary mixture. The Rayleigh and Brillouin lines for the mixture in a steady-state subject to a large, linear temperature gradient are considered. Consistency of the averaged evolution equations requires that the temperature gradient induce a concentration gradient (and vice versa). Mode-coupling theory provides a powerful method whereby a careful study of the nonequilibrium effects in the fluid is possible, since it is possible to deter- mine the relative strengths of the various expansion parameters at all stages of the calculation. This work extends the results of a previous paper ${ }^{12}$ where the theory of fluctuating hydrodynamics was used to study the Rayleigh line in the same system.

\section{LIGHT-SCATTERING SPECTRUM}

The light-scattering spectrum is computed from the dynamic structure factor which is defined in terms of fluctuations of the local dielectric constant $\epsilon(\mathbf{r}, t)$. Experimentally, $\epsilon$ is taken to be a function of the position $\mathbf{r}$ and time $t$ through the temperature $T$, pressure $p$, and concentration $c$. Fluctuations in $\epsilon(\mathbf{r}, t)$ are then computed from the expansion ${ }^{13,14}$

$$
\begin{aligned}
\delta \epsilon(\mathbf{r}, t)= & \left(\frac{\partial \epsilon}{\partial T}\right)_{p, c} \delta T(\mathbf{r}, t)+\left(\frac{\partial \epsilon}{\partial c}\right)_{T, p} \delta c(\mathbf{r}, t) \\
& \left.+\mid \frac{\partial \epsilon}{\partial p}\right)_{T, c} \delta p(\mathbf{r}, t) .
\end{aligned}
$$

In terms of the mode-coupling theory developed in later sections, it is more convenient to expand $\epsilon$ in terms of the variables $\left(s^{\prime}, c, p\right)$ (Refs. 13-15) where $s^{\prime}$ is the modified entropy variable. Local fluctuations in $s^{\prime}$ are defined by

$$
\begin{aligned}
\delta s^{\prime}(\mathbf{r}, t) & =\delta s(\mathbf{r}, t)-\left(\frac{\partial s}{\partial c}\right)_{p, T} \delta c(\mathbf{r}, t) \\
& \left.=\frac{C_{p, c}}{T} \mid \delta T(\mathbf{r}, t)-\frac{\alpha_{T} T}{\rho C_{c, p}} \delta p(\mathbf{r}, t)\right) .
\end{aligned}
$$

Here $s$ is the entropy per unit mass, $\alpha_{T}=-(\partial \rho / \partial T)_{p, c} / \rho$ is the thermal expansion coefficient, and $C_{p, c}=T(\partial s / \partial T)_{p, c}$ is the specific heat (per unit mass) at constant pressure and concentration. Precise definitions of the fluctuating variables are given in Sec. III.

The two expansions are almost equivalent since expan- 
sion (2.2) and the expansion in terms of $s^{\prime}, c$, and $p$ are related by the equations ${ }^{15}$

$$
\begin{aligned}
& \left(\frac{\partial \epsilon}{\partial c}\right)_{p, s^{\prime}}=\left(\frac{\partial \epsilon}{\partial c}\right)_{p, T}, \\
& \left(\frac{\partial \epsilon}{\partial s^{\prime}}\right)_{p, c}=\frac{C_{p, c}}{T}\left(\frac{\partial \epsilon}{\partial c}\right)_{p, T},
\end{aligned}
$$

$$
S(\mathbf{k}, \omega)=\frac{1}{T}\left(\int_{V} d \mathbf{r} P(\mathbf{r})^{2}\right)^{-1} \int_{V} d \mathbf{r}_{1} P\left(\mathbf{r}_{1}\right) \int_{V} d \mathbf{r}_{2} P\left(\mathbf{r}_{2}\right) \int_{-T / 2}^{T / 2} d t_{1} \int_{-T / 2}^{T / 2} d t_{2} e^{-i \mathbf{k} \cdot\left(\mathbf{r}_{1}-\mathbf{r}_{2}\right)-t \omega\left(t_{1}-t_{2}\right)}\left\langle\delta \epsilon\left(\mathbf{r}_{1}, t_{1}\right) \delta \epsilon\left(\mathbf{r}_{2}, t_{2}\right)\right\rangle
$$

where $\hbar \mathbf{k}=\hbar\left(\mathbf{k}_{f}-\mathbf{k}_{l}\right)$ is the momentum transferred by the fluid fluctuations to a photon with an incident wave vector $\mathbf{k}_{i}$ and final wave vector $\mathbf{k}_{f}$, and $\hbar \omega=\hbar\left(\omega_{f}-\omega_{i}\right)$ is the energy transferred to the photon. $V$ is the volume of the fluid, $T$ is the duration of the experiment, and $P(\mathbf{r})$ is a form factor describing the portion of the fluid illuminated during the experiment. The subsequent sections are devoted to calculating the correlation function

\section{$\left\langle\delta \epsilon\left(\mathbf{r}_{1}, t_{1}\right) \delta \epsilon\left(\mathbf{r}_{2}, t_{2}\right)\right\rangle$. \\ III. HYDRODYNAMIC EQUATIONS FOR CORRELATION FUNCTIONS}

The natural variables describing microscopic fluid motion are the conserved fluid densities, denoted by $a_{\alpha}(\mathbf{r}, t)$. The conserved quantities are the mass density of each component,

$$
\rho_{J}(\mathbf{r}, t)=\sum_{l=1}^{N_{J}} \delta\left(\mathbf{r}-\mathbf{r}_{l}(t)\right), \quad j=1,2
$$

the total momentum density

$$
\mathbf{g}(\mathbf{r}, t)=\sum_{i=1}^{N} \mathbf{p}_{t}(t) \delta\left(\mathbf{r}-\mathbf{r}_{i}(t)\right)
$$

the momentum density of each component

$$
\mathbf{g}_{j}(r, t)=\sum_{i=1}^{N_{J}} \mathbf{p}_{i}(t) \delta\left(\mathbf{r}-\mathbf{r}_{i}(t)\right),
$$

and the energy density

$$
\varepsilon(\mathbf{r}, t)=\sum_{i=1}^{N} e_{i}(t) \delta\left(\mathbf{r}-\mathbf{r}_{t}(t)\right),
$$

where $\mathbf{r}_{l}$ and $\mathbf{p}_{i}$ denote the position and momentum of the $i$ th particle. The energy of the $i$ th particle is given by

$$
e_{i}(t)=p_{i}^{2} / 2 m_{i}+\frac{1}{2} \sum_{\substack{j=1 \\ j \neq i}}^{N} \phi\left(\mathbf{r}_{i j}(t)\right) .
$$

Here $N=N_{1}+N_{2}$ is the total number of particles in the

$$
\left(\frac{\partial \epsilon}{\partial p}\right)_{c, s^{\prime}}=\left(\frac{\partial \epsilon}{\partial p}\right)_{c, T}+\frac{\alpha_{T} T}{\rho C_{p, c}}\left(\frac{\partial \epsilon}{\partial T}\right)_{p, c} .
$$

The dynamic structure factor $S(k, \omega)$ is calculated from the time-dependent correlation function

$$
\left\langle\delta \epsilon\left(\mathbf{r}_{1}, t_{1}\right) \delta \epsilon\left(\mathbf{r}_{2}, t_{2}\right)\right\rangle
$$

by the expression ${ }^{2,4}$ system, $\phi$ is the interparticle potential, and $\mathbf{r}_{i j}=\mathbf{r}_{i}-\mathbf{r}_{j}$. The fluctuating quantities are defined by

$$
\delta a_{\alpha}(\mathbf{r}, t)=a_{\alpha}(\mathbf{r}, t)-\left\langle a_{\alpha}(\mathbf{r}, t)\right\rangle_{N}
$$

and the correlation function by

$$
M_{\alpha \beta}\left(\mathbf{r}_{1}, t_{1}, \mathbf{r}_{2}, t_{2}\right) \equiv\left\langle\delta a_{\alpha}\left(\mathbf{r}_{1}, t_{1}\right) \delta a_{\beta}\left(\mathbf{r}_{2}, t_{2}\right)\right\rangle_{N},
$$

where the average \langle\rangle$_{N}$ is defined by

$$
\left\langle f\left(\mathbf{r}, x^{N}, t\right)\right\rangle_{N} \equiv \int d x^{N} F_{N}^{\mathrm{SS}}\left(x^{N}\right) f\left(\mathbf{r}, x^{N}, t\right) .
$$

Here $F_{N}^{\mathrm{SS}}\left(x^{N}\right)$ is the $N$-particle nonequilibrium, steadystate distribution function; $x^{N}$ denotes the phase-space variable describing the system; and $d x^{N}$ denotes the corresponding phase-space element as well as the sum over particles appropriate to a grand canonical ensemble for a binary mixture. In a steady state $\left\langle a_{\alpha}(\mathbf{r}, t)\right\rangle_{N}$ is time independent and

$$
M_{\alpha \beta}\left(\mathbf{r}_{1}, t_{1}, \mathbf{r}_{2}, t_{2}\right)=M_{\alpha \beta}\left(\mathbf{r}_{1}, t_{1}-t_{2}, \mathbf{r}_{2}, 0\right) .
$$

Following Kirkpatrick et al. ${ }^{2,3}$ we assume that the system is sufficiently close to the local equilibrium steady state that an expansion in terms of the gradients can be made, namely,

$$
F_{N}^{\mathrm{SS}}\left(x^{N}\right)=F_{L}^{\mathrm{SS}}\left(x^{N}\right)+F_{\nabla}^{\mathrm{SS}}\left(x^{N}\right) .
$$

Here $F_{L}^{\mathrm{SS}}\left(x^{N}\right)$ denotes the local equilibrium distribution function, given by

$$
F_{L}^{\mathrm{SS}}\left(x^{v}\right)=\frac{e^{y_{\alpha} *_{a}}}{\int d x^{N} e^{y_{\alpha} *_{a}}},
$$

where

$$
y_{\alpha} * a_{\alpha} \equiv \int d \mathbf{r} y_{\alpha}(\mathbf{r}) a_{\alpha}(\mathbf{r})
$$

and $F_{\nabla}^{\mathrm{SS}}\left(x^{N}\right)$ denotes the higher-order corrections to $F_{L}^{\mathrm{SS}}\left(x^{N}\right)$ in terms of an expansion powers of the spatial derivatives of the hydrodynamical variables. The assumption underlying this expansion is that the length scale characterizing the variation of microscopic motion $l$ is much smaller than the correlation length $L$ of the fluctuating quantities. It can be shown that the expansion is 
in powers of $l / L{ }^{3}$ The variables conjugate to the set $\left\{a_{\alpha}\right\}=\left\{\rho_{\alpha}, \mathbf{g}, \varepsilon\right\}$ are denoted by

$$
\left\{y_{\alpha}\right\}=\left\{\beta\left(\mu_{\alpha} / m_{\alpha}-u^{2} / 2\right), \beta \mathbf{u},-\beta\right\},
$$

where $\mu_{\alpha}$ is the chemical potential of the mixture component $\alpha$ and $\mathbf{u}=\langle\mathbf{g}\rangle /\langle\rho\rangle$ is the average velocity. The total density is given by

$$
\rho(\mathbf{r}, t)=\rho_{1}(\mathbf{r}, t)+\rho_{2}(\mathbf{r}, t) .
$$

Consistent with the expansion in powers of $l / L$, the convolution $y_{\alpha} * a_{\alpha}$ appearing in (3.10) can be approximated by $y_{\alpha}(\mathbf{r}) a_{\alpha T}$, where

$$
a_{\alpha T}\left(x^{N}\right)=\int d \mathbf{r} a_{\alpha}\left(\mathbf{r}, x^{N}\right) .
$$

The local equilibrium distribution function $F_{L}^{\mathrm{SS}}$ is replaced by an equilibriumlike distribution function,

$$
F_{0}\left(\mathbf{r}, x^{N}\right)=\frac{e^{y_{\alpha}\langle\mathbf{r}) a_{\alpha} T}}{\int d x^{N} e^{y_{\alpha}(\mathbf{r}) a_{\alpha l}}}
$$

and nonequilibrium averages \langle\rangle$_{N}$ are replaced by averages weighted by $F_{0}$, denoted by \langle\rangle .

Fluctuations in the arbitrary quantity $a(r, t)$ are now given by

$$
\begin{aligned}
\delta a(\mathbf{r}, t)= & \sum_{i=1}^{2}\left(\frac{\partial\langle a\rangle}{\partial\left\langle\rho_{l}\right\rangle}\right) \delta \rho_{i}(\mathbf{r}, t)+\left(\frac{\partial\langle a\rangle}{\partial\langle\mathbf{g}\rangle}\right) \cdot \delta \mathbf{g}(\mathbf{r}, t) \\
& +\left(\frac{\partial\langle a\rangle}{\partial\langle\varepsilon\rangle}\right) \delta \varepsilon(\mathbf{r}, t) .
\end{aligned}
$$

Since the concentration and total density are natural variables to use in mode-coupling theory, it is convenient to replace $\rho_{1}$ and $\rho_{2}$ in the above expansion by $\rho$ and $c$. The concentration $c$ is defined by

$$
c(\mathbf{r}, t)=\rho_{1}(\mathbf{r}, t) / \rho(\mathbf{r}, t),
$$

and fluctuations in $c$ are given by

$$
\delta c(\mathbf{r}, t)=\frac{\left\langle\rho_{2}\right\rangle}{\langle\rho\rangle^{2}} \delta \rho_{1}(\mathbf{r}, t)-\frac{\left\langle\rho_{1}\right\rangle}{\langle\rho\rangle^{2}} \delta \rho_{2}(\mathbf{r}, t) .
$$

Following the projection-operator techniques employed by Kirkpatrick et $a l .^{2}$ or alternatively employing equations from fluctuating hydrodynamics, ${ }^{16}$ we obtain the following evolution equations for the correlation functions $M_{\alpha \beta} \equiv M_{\alpha \beta}\left(\mathbf{r}_{1}, \mathbf{r}_{2}, t\right)$ :

$$
\begin{aligned}
& \frac{\partial}{\partial t} M_{\rho \beta}+\frac{\partial}{\partial r_{1 \imath}} M_{g_{\imath} \beta}=0 \\
& \frac{\partial}{\partial t} M_{g_{l} \beta}=-\frac{\partial}{\partial r_{1 j}}\left[\delta_{\imath \jmath}\left(\frac{\partial p}{\partial \rho^{\alpha}}\right) M_{\rho^{\alpha} \beta}+\delta_{l j}\left(\frac{\partial p}{\partial \varepsilon}\right) M_{\varepsilon \beta}+u_{\jmath} M_{g_{l} \beta}+g_{l} M_{u_{j} \beta}-\eta \Delta_{l \jmath, k l} \frac{\partial}{\partial r_{1 k}} M_{u_{l} \beta}-\zeta \frac{\partial}{\partial r_{1 k}} M_{u_{k} \beta}\right. \\
& \left.+M_{\eta \beta} \Delta_{l \jmath, k l} \frac{\partial}{\partial r_{1 k}} u_{l}-M_{\varsigma \beta} \frac{\partial}{\partial r_{1 k}} u_{k}\right] \\
& \rho \frac{\partial}{\partial t} M_{c \beta}+M_{g_{\imath} \beta} \frac{\partial c}{\partial r_{1 \iota}}=\frac{\partial}{\partial r_{1 \iota}}\left[\rho D\left[\frac{\partial}{\partial r_{1 \iota}} M_{c \beta}+\frac{k_{I}}{T} \frac{\partial}{\partial r_{1 \imath}} M_{T \beta}+\frac{k_{p}}{p} \frac{\partial}{\partial r_{1 \iota}} M_{p \beta}+M_{\left(k_{T} / T\right) \beta} \frac{\partial T}{\partial r_{1 \iota}}\right]\right], \\
& \frac{\partial}{\partial t} M_{\varepsilon \beta}+u_{\imath} \frac{\partial}{\partial r_{1 \imath}} M_{\varepsilon \beta}+M_{u_{\imath} \beta} \frac{\partial \varepsilon}{\partial r_{1 l}}+\hat{h} \frac{\partial}{\partial r_{1 \imath}} M_{u_{\imath} \beta}+M_{h \beta} \frac{\partial}{\partial r_{1 l}} u_{i} \\
& =2\left(\eta \Delta_{l j, k l} \frac{\partial}{\partial r_{k}} u_{l}+\zeta \frac{\partial}{\partial r_{k}} u_{k}\right) \frac{\partial}{\partial r_{l}} M_{u_{j} \beta}+M_{\eta \beta} \Delta_{l j, k l} \frac{\partial}{\partial r_{1 k}} u_{l} \frac{\partial}{\partial r_{1 l}} u_{j}+M_{\zeta \beta}\left(\frac{\partial}{\partial r_{1 k}} u_{l}\right)^{2} \\
& +\frac{\partial}{\partial r_{1 \iota}} \int M_{D_{T} \beta} \frac{\partial T}{\partial r_{1 \iota}}+D_{T} \frac{\partial}{\partial r_{1 i}} M_{T \beta}+M_{, \beta} \frac{\partial c}{\partial r_{1 \iota}}+M_{\left(, k_{T} / T\right) \beta} \frac{\partial T}{\partial r_{1 \iota}} \\
& \left.+M_{\left(r k_{p} / p\right) \beta} \frac{\partial p}{\partial r_{1 \iota}}+d \frac{\partial}{\partial r_{1 \iota}} M_{c \beta}+\frac{d k_{T}}{T} \frac{\partial}{\partial r_{1 \iota}} M_{T \beta}+\frac{d k_{p}}{p} \frac{\partial}{\partial r_{1 \iota}} M_{p \beta}\right) \text {. }
\end{aligned}
$$

The repeated index convention is used to denote summation. Throughout the paper we employ the convention that greek letter indices denote the conserved densities $\{\rho, c, \mathbf{g}, \varepsilon\}$ and roman letter indices, commencing with the letter $i,\{i, j, \ldots\}$ denote vector components. Further,

$$
\begin{aligned}
& \Delta_{l \jmath, k l}=\delta_{l k} \delta_{\jmath l}+\delta_{l l} \delta_{\jmath k}-\frac{2}{3} \delta_{l j} \delta_{k l}, \\
& d \equiv \rho \xi D
\end{aligned}
$$

$$
\xi \equiv k_{T}\left(\frac{\partial \mu}{\partial c}\right)_{p, \Gamma}-T\left(\frac{\partial \mu}{\partial T}\right)_{p, c}+\mu .
$$

Here $\rho, c, \mathbf{u}, \mathbf{g}, \varepsilon, p, \hat{h}$, and $\mu$ are the averaged density, concentration, velocity, momentum density, energy density, pressure, enthalpy density, and chemical potential at the point $r$. The transport coefficients, also functions of position $\mathbf{r}$, are the thermal conductivity $\lambda$, the kinematic 
viscosity $v$, the thermal diffusivity $D_{T}=\lambda / \rho C_{p, c}$, the mass diffusivity $D$, the thermal diffusion ratio $k_{T}$, and the barodiffusion ratio $k_{p}$.

Quantities of the form $\mathcal{M}_{(x) \beta}$ are defined by

$$
M_{(x) \beta}=\left(\frac{\partial x}{\partial \rho}\right)_{\varepsilon, c} M_{\rho \beta}+\left(\frac{\partial x}{\partial c}\right)_{\rho, \varepsilon} M_{c \beta}+\left(\frac{\partial x}{\partial \varepsilon}\right)_{\rho, c} M_{\varepsilon \beta} .
$$

Further,

$$
\rho M_{u_{1} \beta}=M_{g_{l} \beta}-u_{i} M_{\rho \beta} .
$$

The evolution equations (3.17)-(3.20) can be written more concisely in the form

$$
\frac{\partial}{\partial t} M_{\alpha \beta}\left(\mathbf{r}_{1}, \mathbf{r}_{2}, t\right)=H_{\alpha \mu}\left(\mathbf{r}_{1}\right) M_{\mu \beta}\left(\mathbf{r}_{1}, \mathbf{r}_{2}, t\right),
$$

where $H_{\alpha \mu}\left(\mathbf{r}_{1}\right)$ is defined by Eqs. (3.17)-(3.20).

\section{STEADY-STATE EQUATIONS}

The evolution equations (3.17)-(3.20) apply for arbitrary functional forms of the steady-state velocity, concentration, density, temperature, and pressure. However, since the effect of a velocity gradient is substantially smaller than that of a temperature gradient (or concentration gradient) by a factor proportional to the speed of sound, ${ }^{2}$ we consider steady-state conditions for which the mean velocity

$$
\mathbf{u}=\mathbf{0}
$$

and the temperature varies linearly in one direction, chosen here to be the $x$ direction. The equations for steady-state mean flow then require that

$$
\begin{aligned}
& \frac{\partial}{\partial r_{1 x}} c=-\frac{k_{T}}{T} \frac{\partial}{\partial r_{1 x}} T, \\
& \frac{\partial}{\partial \mathbf{r}_{1}} p=0 .
\end{aligned}
$$

The relevant expansion parameter is the inverse length scale of the temperature gradient, defined by

$$
X^{T} \equiv \frac{1}{T} \frac{\partial}{\partial r_{1 x}} T
$$

\section{GRADIENT EXPANSIONS}

In equilibrium the correlation function $M_{\alpha \beta}\left(\mathbf{r}_{1}, \mathbf{r}_{2}, t\right)$ is a function of the relative coordinate $\mathbf{r}_{12}=\mathbf{r}_{2}-\mathbf{r}_{1}$ only. However, due to the temperature and concentration gradients, the system is not translationally invariant, and $M_{\alpha \beta}$ is a function of both $\mathbf{r}_{1}$ and $\mathbf{r}_{2}$. Due to the form of the expression (2.4) for $S(\mathbf{k}, \omega)$, it is convenient to transform to the center-of-mass coordinate $\mathbf{r}=\left(\mathbf{r}_{1}+\mathbf{r}_{2}\right) / 2$ and relative coordinate $\mathbf{r}_{12}$ and then introduce the Fourier transform with respect to $\mathbf{r}_{12}$ and the Laplace transform with respect to $t$, where

$$
M_{\alpha \beta}(\mathbf{r}, \mathbf{q}, z)=\int d \mathbf{r}_{12} \int_{0}^{\infty} d t e^{-z t-i \mathbf{q} \cdot \mathbf{r}_{12}} M_{\alpha \beta}\left(\mathbf{r}, \mathbf{r}_{12}, t\right)
$$

The Fourier and Laplace transforms are applied to the evolution equation (3.23) by first expressing $H_{\alpha \mu}\left(\mathbf{r}_{1}\right)$ in terms of $\mathbf{r}$ and $\mathbf{r}_{12}$ and expanding $H_{\alpha \mu}\left(\mathbf{r}, \mathbf{r}_{12}\right)$ in terms of the gradients. The resulting equations can be written in the form

$$
\begin{array}{r}
z M_{\alpha \beta}(\mathbf{r}, \mathbf{q}, z)-\left[H_{\alpha \mu}^{0}(\mathbf{r}, \mathbf{q})+H_{\alpha \mu}^{1}(\mathbf{r}, \mathbf{q})\right] M_{\mu \beta}(\mathbf{r}, \mathbf{q}, z) \\
=M_{\alpha \beta}(\mathbf{r}, \mathbf{q}) .
\end{array}
$$

The elements $H_{\alpha \mu}^{0}(\mathbf{r}, \mathbf{q})$ are the gradient-independent terms and the elements $H_{\alpha \mu}^{1}(\mathbf{r}, \mathbf{q})$ contain first and higher derivatives in $T$ and $c . M_{\alpha \beta}(\mathbf{r}, \mathbf{q})$ denotes the equal-time correlation function $M_{\alpha \beta}(\mathbf{r}, \mathbf{q}, t=0)$. The equal-time correlation functions are discussed at length in Sec. VI. For the binary mixture the nonzero elements of $H_{\alpha \mu}^{0}(\mathbf{r}, \mathbf{q})$ are

$$
\begin{aligned}
& H_{\rho g_{l}}^{0}=-i q_{i} \\
& H_{c \rho}^{0}=-q^{2}\left[A_{4} \frac{D k_{T}}{T}+A_{1} \frac{D k_{p}}{p}\right] \text {, } \\
& H_{c \varepsilon}^{0}=-q^{2}\left(A_{5} \frac{D k_{T}}{T}+A_{2} \frac{D k_{p}}{p}\right) \text {, } \\
& H_{c c}^{0}=-q^{2}\left(A_{4}^{\prime} \frac{D k_{T}}{T}+A_{1}^{\prime} \frac{D k_{p}}{p}+D\right), \\
& H_{g_{1} \rho}^{0}=-i q_{l} A_{1}, \quad H_{g_{l} c}^{0}=-i q_{i} A_{1}^{\prime}, \quad H_{g_{1} \varepsilon}^{0}=-i q_{1} A_{2} \text {, } \\
& H_{g_{l} g_{j}}^{0}=-v q^{2} \delta_{i j}-q_{\imath} q_{J}\left(\frac{1}{3} v+\frac{\xi}{\rho}\right), \\
& H_{\varepsilon g_{l}}^{0}=-i q A_{3}, \\
& H_{\varepsilon \rho}^{0}=-q^{2}\left[\left(\lambda+\frac{k_{T} d}{T}\right] A_{4}+A_{1} \frac{k_{p} d}{p}\right], \\
& H_{\varepsilon c}^{0}=-q^{2}\left[\left(\lambda+\frac{k_{T} d}{T}\right] A_{4}^{\prime}+A_{1}^{\prime} \frac{k_{p} d}{p}+d\right] \text {, } \\
& H_{\varepsilon \varepsilon}^{0}=-q^{2}\left[\left(\lambda+\frac{k_{T} d}{T}\right) A_{5}+A_{2} \frac{k_{p} d}{p}\right],
\end{aligned}
$$

where

$$
\begin{aligned}
& A_{1}=\left(\frac{\partial p}{\partial \rho}\right)_{\varepsilon, c}, \quad A_{1}^{\prime}=\left(\frac{\partial p}{\partial c}\right)_{\varepsilon, \rho}, \\
& A_{2}=\left(\frac{\partial p}{\partial \varepsilon}\right)_{\rho, c}=\frac{c_{s}^{2} \alpha_{T}}{C_{p, c}}, \\
& A_{3}=\left(\frac{\partial \varepsilon}{\partial \rho}\right)_{s, c}=\hat{h}, \quad A_{3}^{\prime}=\left(\frac{\partial p}{\partial c}\right)_{s, \rho}=\rho \mu, \\
& A_{4}=\left(\frac{\partial T}{\partial \rho}\right)_{\varepsilon, c}, \quad A_{4}^{\prime}=\left(\frac{\partial T}{\partial c}\right)_{\varepsilon, \rho}, A_{5}=\left(\frac{\partial T}{\partial \varepsilon}\right)_{\rho, c} .
\end{aligned}
$$

Here $c_{s}$ is the speed of sound defined by $c_{s}=(\partial p / \partial \rho)_{s, c}$. Note that the quantities $\left\{A_{1}, \ldots, A_{5}\right\}$ as well as the transport coefficients and thermodynamic quantities ap- 
pearing in the equations for $H^{0}$ all depend on the position r.

In this paper we consider large gradients, namely, gradients sufficiently large that the time scale of the temperature and concentration gradient $1 /\left(c_{s} X^{T}\right)$ is of the order of the decay time scale of the fluctuating densities $1 /\left(\Gamma_{s} q^{2}\right)$. Although $\Gamma_{s}$ is the sound damping constant, from its definition, Eq. (B4), we note that it can be used to characterize the decay rate of all fluctuations. Since $\Gamma_{s} / c_{s} \simeq l$ and we require for large gradients that

$$
c_{s} X^{T} / \Gamma_{s} q^{2} \simeq X^{T} / l q^{2} \simeq 1,
$$

it follows from the hydrodynamic conditions, namely, $l / L \simeq l q \ll<1$, that $X^{T} / q \ll<1$. Further, since $\Gamma_{s} / c_{s} \simeq l$, the ratio of the widths of the peaks in the scattering spectrum to the distance between the Rayleigh peak and Brillouin peaks, namely, $\Gamma_{s} q^{2} / c_{s} q \simeq l q<<1$. Hence under these conditions the peaks remain well separated.

That the above requirements are realistic can be seen by comparing the estimates with experimental values ${ }^{11,12}$ of $q, X^{T}, c_{s}$, and $\Gamma_{s}$ obtained with one-component fluids. Typical values are $q \simeq 2000 \mathrm{~cm}^{-1}, X^{T} \simeq 0.5 \mathrm{~cm}^{-1}$, $c_{s} \simeq 3 \times 10^{4} \mathrm{~cm} / \mathrm{s}$, and $\Gamma_{\mathrm{s}} \simeq 10^{-2} \mathrm{~cm}^{2} / \mathrm{s}$ for which $c_{s} X^{T} / \Gamma_{s} q^{2} \simeq 1$ and the relation $X^{T} / q \ll<1$ is well satisfied.

An examination of the structure $H_{\alpha \mu}^{1}(\mathbf{r}, \mathbf{q})$ from Eqs. (3.17)-(3.20) reveals that $H_{\alpha \mu}^{1}(\mathbf{r}, \mathbf{q})$ can be expanded as

$$
H_{\alpha \mu}^{1}(\mathbf{r}, \mathbf{q})=c_{\varsigma} X^{T}\left[1+O\left(X^{T} / q\right)\right][1+O(l q)] .
$$

According to the above discussion, we neglect terms of $O\left(X^{T} / q\right)$ relative to terms of $O\left(X^{T} / q^{2}\right)$. The solutions $M_{\alpha \beta}(\mathbf{r}, \mathbf{q}, z)$ [and $M_{\alpha \beta}(\mathbf{r}, \mathbf{q})$ ] then obtained by iteration ${ }^{4,5}$ have the form

$$
\begin{aligned}
& M_{\alpha \beta}(\mathbf{r}, \mathbf{q}, z) \\
& \quad=\left[O\left(X^{T} / q^{2}\right)+O\left(X^{T} / q^{2}\right)^{2}+\cdots\right][1+O(l q)]
\end{aligned}
$$

All the terms of the form $\left(X^{T} / q^{2}\right)^{n}$ are due to the leading term $c_{s} X^{T}$ in $H_{\alpha \mu}^{1}(\mathbf{r}, \mathbf{q})$. Consequently, the dominant terms in $M_{\alpha \beta}(\mathbf{r}, \mathbf{q}, z)$ are retained when only this leading term in $H_{\alpha \mu}^{1}(\mathbf{r}, \mathbf{q})$ is kept. Solutions of $M_{\alpha \beta}(\mathbf{r}, \mathbf{q}, z)$ and $M_{\alpha \beta}(\mathbf{r}, \mathbf{q})$ to all orders in $\left(X^{T} / q^{2}\right)$ are thus obtained by solving Eqs. (5.2) [and later also (6.1) for the equal-time correlation functions] with all the terms in $H_{\alpha \mu}^{1}(\mathbf{r}, \mathbf{q})$, except those of $O\left(X^{T} q^{0}\right)$, neglected.

The nonzero elements of $H_{\alpha \mu}^{1}(\mathbf{r}, \mathbf{q})$ to $O\left(X^{T} q^{0}\right)$ are

$$
\begin{aligned}
& H_{\rho g_{l}}^{1}=-\frac{1}{2} \delta_{l x} \frac{\partial}{\partial r_{x}}, \\
& H_{c g_{l}}^{1}=\frac{k_{T}}{\rho} \delta_{l x} X^{T}, \\
& H_{g_{l} \rho}^{1}=-\frac{1}{2} \delta_{l x} \frac{\partial}{\partial r_{x}} A_{1}+\frac{1}{2} q_{l} \delta_{l x}\left(\frac{\partial A_{1}}{\partial r_{x}}\right) \frac{\partial}{\partial q_{x}}, \\
& H_{g_{l} c}^{1}=-\frac{1}{2} \delta_{l x} \frac{\partial}{\partial r_{x}} A_{1}^{\prime}+\frac{1}{2} q_{l} \delta_{l x}\left(\frac{\partial A_{1}^{\prime}}{\partial r_{x}}\right) \frac{\partial}{\partial q_{x}}, \\
& H_{g_{l} \varepsilon}^{1}=-\frac{1}{2} \delta_{l x} \frac{\partial}{\partial r_{x}} A_{2}+\frac{1}{2} q_{l} \delta_{l x}\left(\frac{\partial A_{2}}{\partial r_{x}}\right) \frac{\partial}{\partial q_{x}},
\end{aligned}
$$

$$
H_{\varepsilon g_{l}}^{1}=-\frac{1}{2} \delta_{l x} \frac{\partial}{\partial r_{x}} A_{3}+\frac{1}{2} q_{i} \delta_{l x}\left(\frac{\partial A_{3}}{\partial r_{x}}\right) \frac{\partial}{\partial q_{x}} .
$$

\section{EQUAL-TIME CORRELATIONS}

The equal-time correlation function elements $M_{\alpha \beta}(\mathbf{r}, \mathbf{q})$ are separated into two parts,

$$
M_{\alpha \beta}(\mathbf{r}, \mathbf{q})=A_{\alpha \beta}(\mathbf{r}, \mathbf{q})+D_{\alpha \beta}(\mathbf{r}, \mathbf{q}),
$$

where the elements $A_{\alpha \beta}(\mathbf{r}, \mathbf{q})$ are independent of $X^{T}$ and $D_{\alpha \beta}(\mathbf{r}, \mathbf{q})$ contains terms to all orders in $X^{T} / q^{2}$. The elements $A_{\alpha \beta}(\mathbf{r}, \mathbf{q})$ are calculated straightforwardly from the definition

$$
A_{\alpha \beta}(\mathbf{r}, \mathbf{q})=\left(\delta a_{\alpha}(\mathbf{q}), \delta a_{\beta}(-\mathbf{q})\right)
$$

and results from equilibrium thermodynamics of fluctuations. ${ }^{13}$ In Eq. (6.2) the scalar product of arbitrary functions $f(\mathbf{q})$ and $g(\mathbf{q})$ is defined by

$$
(f(\mathbf{q}), g(\mathbf{q}))=\langle f(\mathbf{q}) g(\mathbf{q})\rangle / V,
$$

where $V$ is the volume of the system. The $\mathbf{r}$ dependence in $A_{\alpha \beta}(\mathbf{r}, \mathbf{q})$ is due to the position dependence of distribution function $F_{0}$ in the averaging process. The explicit values for $A_{\alpha \beta}(\mathbf{r}, \mathbf{q})$ are given in Appendix A.

The procedure to obtain the elements $D_{\alpha \beta}(\mathbf{r}, \mathbf{q})$ is more complicated. Following the method of Ernst et al. ${ }^{17}$ or Kirkpatrick et al. ${ }^{4,5}$ where the exact Liouville timeevolution operators are replaced by coarse-grained timeevolution operators, we obtain the following equations for $D_{\alpha \beta}(\mathbf{r}, \mathbf{q})$,

$$
\begin{aligned}
H_{\alpha \mu}(\mathbf{r}, \mathbf{q}) D_{\mu \beta}(\mathbf{r}, \mathbf{q})+H_{\beta \mu}(\mathbf{r},-\mathbf{q}) D_{\alpha \mu}(\mathbf{r}, \mathbf{q}) \\
\quad=\left(\delta a_{\alpha}(\hat{\mathbf{q}}) \delta a_{\beta}(-\hat{\mathbf{q}}) I_{v, l T}\right) \frac{\partial}{\partial r_{l}} y_{v}(\mathbf{r}) .
\end{aligned}
$$

Here

$$
I_{v, l T}=\int_{V} d \mathbf{r} I_{v, l}(\mathbf{r}, t)
$$

and $I_{v, t}(\mathrm{r}, t)$ is the projected flux defined by

$$
I_{v, t}(\mathbf{r}, t)=(1-P) \delta J_{v, i}(\mathbf{r}, t)
$$

and $J_{v, l}(\mathbf{r})$ is a current defined by

$$
\frac{d}{d t} a_{v}(\mathbf{r}, t)=-\frac{\partial}{\partial r_{l}} J_{v, l}(\mathbf{r}, t) .
$$

The projection operator ${ }^{2} 17 P$ projects variables onto space spanned by the conserved densities. Explicit expressions for the various fluxes are given in Appendix B.

Solutions for $D_{\alpha \beta}(\mathbf{r}, \mathbf{q})$ are obtained by expanding Eq. (6.3) in terms of the eigenvectors of $H_{\alpha \beta}^{0}$. The eigenvectors, eigenvalues, and modes are discussed in Appendix B. For a binary mixture there are six modes, namely, two sound modes traveling in opposite directions, denoted by subscript $\sigma, \sigma= \pm 1$, two viscous modes directed perpendicular to the sound modes, denoted by $\eta_{l}, i=1,2$, a concentration mode denoted by subscript $c$, and a heat (or modified entropy) mode, denoted by the subscript $H$.

The elements of $H$ and $D$ in terms of the eigenvectors are defined by

$$
H_{a b} \equiv \theta_{a}^{L}(\widehat{\mathbf{q}}) \cdot H \cdot \theta_{b}^{R}(\widehat{\mathbf{q}}),
$$




$$
D_{a b}=\theta_{a}^{L}(\hat{\mathbf{q}}) \cdot D \cdot \widetilde{\theta}_{b}^{L}(-\hat{\mathbf{q}}),
$$

where $\theta_{a}^{L}(\widehat{\mathbf{q}})$ and $\theta_{a}^{R}(\widehat{\mathbf{q}})$ are the left and right eigenvectors of $H^{0} . \widetilde{\theta}_{b}^{L}(-\hat{\mathbf{q}})$ is the transposed matrix of $\theta_{b}^{L}(-\hat{\mathbf{q}})$ and the dot product indicates matrix multiplication. Roman letter subscripts $a, b, \ldots$ will be used to denote elements of the set of modes $\left\{ \pm \sigma, c, \eta_{l}, H\right\}$. Equation (6.3) becomes

$$
\begin{array}{r}
H_{a d}^{0} D_{d b}+H_{b d}^{0}(-q) D_{a d}+H_{a d}^{1} D_{d b}+H_{b d}^{1}(-q) D_{a d} \\
=\left(\delta a_{a}(\widehat{\mathbf{q}}) \delta a_{b}(-\hat{\mathbf{q}}) \mathbf{S}_{x}\right) \beta X^{T},
\end{array}
$$

where the obvious arguments in $\mathbf{q}$ and $\mathbf{r}$ have been neglected. The flux $\mathbf{S}_{x}$ is given in Appendix B. If $a$ and $b$ denote either a sound or viscous mode,

$$
\begin{aligned}
& H_{a d}^{0}(\mathbf{q})=-\lambda_{a}(\mathbf{q}) \delta_{a d}, \\
& H_{a d}^{0}(-\mathbf{q})=-\lambda_{a}(-\mathbf{q}) \delta_{a d},
\end{aligned}
$$

for any mode $d$. However, the concentration and heat eigenvectors do not diagonalize $H^{0}$. For these cases,

$$
\begin{aligned}
& H_{c d}^{0}=H_{c c}^{0} \delta_{d c}+H_{c H}^{0} \delta_{d H}, \\
& H_{H d}^{0}=H_{H H}^{0} \delta_{d H}+H_{H c}^{0} \delta_{d c},
\end{aligned}
$$

for any $d . H_{H H}^{0}, H_{H c}^{0}, H_{c H}^{0}$, and $H_{c c}^{0}$ are given in Appendix B.

Equation (6.8a) is correct only if $a, b$, and $d$ denote the dissipative modes, namely, $\left\{c, H, \eta_{i}\right\}$. For the sound modes a correction term is needed. The correction term is due to the fact that $H^{1}$ contains differential operators [see Eq. (5.5)] which act on the spatially dependent variables in the eigenvectors when the expansion of Eq. (6.3) is made. The form of the correction terms is discussed in Appendix C. Correction terms that arise for the dissipative modes are shown in Appendix $\mathrm{C}$ to cancel exactly.

An expansion in terms of the modes is convenient since, for the dissipative elements, the only nonzero elements of $H^{1}$ are

$$
\begin{aligned}
& H_{H \eta_{t}}^{1}=c_{s} \alpha_{T} T X^{T} \hat{q}_{\perp x}^{(i)} \delta_{t 1}, \\
& H_{c \eta_{t}}^{1}=c_{s} k_{T} T X^{T} \widehat{q}_{\perp x}^{(i)} \delta_{i 1} / \rho .
\end{aligned}
$$

We note that $H^{1}$ is not symmetric and $H_{\eta, H}=H_{\eta, c}=0$. Here $\widehat{q}_{\perp x}^{(i)}$ is the $x$ component of $\widehat{\mathbf{q}}_{\perp}^{(l)}, i=1,2$ where $\widehat{\mathbf{q}}_{\perp}^{(l)}$ are unit vectors orthogonal to $\hat{\mathbf{q}}$ and each other. $\hat{\mathbf{q}}_{1}^{(1)}$ is constructed so that it does not change sign under reflection of q, namely, ${ }^{4}$

$$
\begin{aligned}
& \hat{\mathbf{q}}_{\perp}^{(1)}=\left(-\hat{q}_{y}^{2}-\hat{q}_{z}^{2}, \hat{q}_{y} \hat{q}_{x}, \hat{q}_{z} \hat{q}_{x}\right)\left(\hat{q}_{y}^{2}+\hat{q}_{z}^{2}\right)^{-1 / 2}, \\
& \hat{\mathbf{q}}_{\perp}^{(2)}=\left(0,-\hat{q}_{z}, \hat{q}_{y}\right)\left(\hat{q}_{y}^{2}+\widehat{q}_{z}^{2}\right)^{-1 / 2} .
\end{aligned}
$$

Since terms of $O\left(X^{T} / q\right)$ are neglected relative to terms of $O\left(X^{T} / q^{2}\right)$ it follows from Eqs. $(6.8 \mathrm{a})-(6.8 \mathrm{c})$ that the equations for $D_{\sigma, \sigma^{\prime}}$, where $\sigma, \sigma^{\prime}$ denote sound modes, decouple from the equations for $D_{a b}$, where $a, b$ denote the dissipative modes. To verify this ${ }^{18}$ we note that the right-hand-side terms in $(6.8 \mathrm{a})$ are proportional to $X^{T}$. Further we have retained only those elements of $H^{1}$ which are $O\left(X^{T}\right)$. For the sake of simplifying this discussion, we replace the terms containing $H^{0}$ in Eq. (6.8a) by $\lambda_{a}(\mathbf{q})+\lambda_{b}(-\mathbf{q})$. (However, the argument is still valid in general.) Thus when $a$ and $b$ denote dissipative modes, then the combination $\lambda_{a}(\mathbf{q})+\lambda_{b}(-\mathbf{q})$ is $O\left(q^{2}\right)$ and consequently the solution $D_{a b}$ contains all powers of $X^{T} / q^{2}$. However, if either $a$ or $b$, but not both, denotes a sound mode then

$$
\lambda_{a}(\mathbf{q})+\lambda_{b}(-\mathbf{q})=O(q)
$$

and the solution for $D_{a b}$ in powers of $O\left(X^{T} / q\right)$ is generated. However, we neglect these terms relative to terms of $O\left(X^{T} / q^{2}\right)$. Thus all the elements coupling the propagating modes to the dissipative modes, for example, $D_{\sigma H}$, are neglected. For the sound-sound terms,

$$
\lambda_{a}(\mathbf{q})+\lambda_{b}(-\mathbf{q})=O\left(q^{2}\right)
$$

only if $a=\sigma$ and $b=-\sigma$. Hence the only sound-sound term not neglected is $D_{\sigma,-\sigma}$.

The correlations

$$
\left(\delta a_{a}(\hat{\mathbf{q}}) \delta a_{b}(-\hat{\mathbf{q}}) \mathbf{S}_{x}\right)
$$

are discussed in Appendix A. The only nonzero correlations are

$$
\begin{aligned}
\left(\delta a_{\eta_{l}}(\hat{\mathbf{q}}) \delta a_{H}(-\hat{\mathbf{q}}) \mathbf{S}_{x T}\right) & =\left(\delta a_{H}(\hat{\mathbf{q}}) \delta a_{\eta_{t}}(-\hat{\mathbf{q}}) \mathbf{S}_{x T}\right) \\
& =-\delta_{l 1} \widehat{q} \stackrel{(1)}{(1)} \frac{\rho \alpha_{T} T}{\beta^{2} c_{s}}, \\
\left(\delta a_{\eta_{l}}(\hat{\mathbf{q}}) \delta a_{c}(-\hat{\mathbf{q}}) \mathbf{S}_{x T}\right) & =\left(\delta a_{c}(\hat{\mathbf{q}}) \delta a_{\eta_{t}}(-\hat{\mathbf{q}}) \mathbf{S}_{x T}\right) \\
& =-\delta_{i 1} \hat{q}_{\perp x}^{(1)} \frac{k_{T}}{\beta^{2} c_{s}} .
\end{aligned}
$$

The resulting equations for dissipative-dissipative equaltime correlations are:

(a) Viscous-viscous correlation.

$$
-2 v q^{2} D_{\eta_{l} \eta_{j}}=0 \text {. }
$$

(b) Viscous-heat and viscous-concentration correlations.

$$
\begin{array}{r}
-v q^{2} D_{\eta_{t} H}+H_{H H}^{0} D_{\eta_{t} H}+H_{H c}^{0} D_{\eta_{l} c}+\delta_{l 1} H_{H \eta_{t}}^{1} D_{\eta_{l} \eta_{l}} \\
=\left(\delta a_{\eta_{t}}(\hat{\mathbf{q}}) \delta a_{H}(-\hat{\mathbf{q}}) \mathbf{S}_{x T}\right) \beta X^{T}, \\
-v q^{2} D_{\eta_{t} c}+H_{c H}^{0} D_{\eta_{t} H}+H_{c c}^{0} D_{\eta_{t} c}+\delta_{l 1} H_{c \eta_{t}}^{1} D_{\eta_{l} \eta_{t}} \\
=\left(\delta a_{\eta_{t}}(\hat{\mathbf{q}}) \delta a_{c}(-\hat{\mathbf{q}}) \mathbf{S}_{x T}\right) \beta X^{T} .
\end{array}
$$

(c) Heat-concentration correlations.

$$
2 H_{H H}^{0} D_{H H}+H_{H c}^{0}\left(D_{c H}+D_{H c}\right)+H_{c \eta_{1}}^{1}\left(D_{\eta_{1} H}+D_{H \eta_{1}}\right)=0 \text {, }
$$

$$
\begin{aligned}
\left(H_{H H}^{0}+H_{c c}^{0}\right) D_{H c}+H_{c H}^{0} D_{H H}+ & H_{H c}^{0} D_{c c}+H_{H \eta_{1}}^{1} D_{\eta_{1} c} \\
& +H_{c \eta_{1}}^{1} D_{H \eta_{1}}=0, \\
2 H_{c c}^{0} D_{c c}+H_{c H}^{0}\left(D_{c H}+D_{H c}\right)+ & H_{H \eta_{1}}^{1}\left(D_{\eta_{1} c}+D_{c \eta_{1}}\right)=0 .
\end{aligned}
$$


The matrix elements $H_{H H}^{0}, H_{c c}^{0}, H_{c H}^{0}$, and $H_{H c}^{0}$ are given in Appendix B. The equations for $D_{c \eta_{t}}$ and $D_{H \eta_{t}}$ are not included since, due to the symmetry of the correlations (6.11a) and $(6.11 \mathrm{~b})$, it follows that $D_{\eta, c}=D_{c \eta_{1}}$ and $D_{\eta_{1} H}=D_{H \eta}$. Consequently, $D_{H c}=D_{c H}$. The solutions for $D_{H \eta_{t}}$ and $D_{c \eta_{t}}$ are

$$
D_{H \eta_{l}}=X^{T} \widehat{q}_{\perp x}^{(1)} \frac{\rho \alpha_{T} T q^{2}(v+D)}{\beta c_{s}\left(v q^{2}+z_{+}\right)\left(v q^{2}+z_{-}\right)}
$$

and

$$
D_{c \eta_{l}}=X^{T} \widehat{q}_{\perp x}^{(1)} \frac{k_{T} q^{2}\left(v+\mathcal{D}+D_{T}\right)}{\beta c_{s}\left(v q^{2}+z_{+}\right)\left(v q^{2}+z_{-}\right)} .
$$

Here we have introduced the variables $\mathcal{D}, z_{+}$, and $z_{-}$, defined by

$$
D=D\left[1+\frac{k_{T}^{2}}{T C_{p, c}}\left(\frac{\partial \mu}{\partial c}\right]_{p, T}\right]
$$

and

$$
z_{ \pm}=\frac{1}{2} q^{2}\left\{D_{T}+\mathcal{D} \pm\left[\left(D_{T}+\mathcal{D}\right)^{2}-4 D_{T} \mathcal{D}\right]^{1 / 2}\right\} .
$$

The solutions for $D_{H H}, D_{c c}, D_{c H}$, and $D_{H c}$ are

$$
D_{H H}=\frac{\rho\left(\alpha_{T} T X^{T} \hat{q}_{1 . x}^{1}\right)^{2}\left[\mathcal{D}^{2}\left(D_{T}+\mathcal{D}+v\right)+D D_{T} v\right]}{\beta D D_{T}(D+D)\left(v q^{2}+z_{+}\right)\left(v q^{2}+z_{-}\right)}
$$

$$
D_{c c}=\frac{\left(k_{T} X^{T} \widehat{q}_{1 x}^{1}\right)^{2}\left[\left(\mathcal{D}+D_{T}\right)^{2}\left(D_{T}+\mathcal{D}+v\right)+D D_{T} v\right]}{\rho \beta D D_{T}(D+D)\left(v q^{2}+z_{+}\right)\left(v q^{2}+z_{-}\right)},
$$

and

$$
D_{H c}=D_{c H}=-\frac{\alpha_{T} k_{T}\left(X^{T} \widehat{q}_{1 x}^{1}\right)^{2}\left[\mathcal{D}\left(\mathcal{D}+D_{T}\right)\left(D_{T}+\mathcal{D}+v\right)+D D_{T} v\right]}{\beta D D_{T}(D+D)\left(v q^{2}+z_{+}\right)\left(v q^{2}+z_{-}\right)} .
$$

Note that, whereas the equal-time correlations which are coupled to the viscous mode $D_{c \eta_{t}}$ and $D_{H \eta_{t}}$ are $O\left(X^{T} / q^{2}\right)$, other dissipative-dissipative equal-time correlations are $O\left(X^{T} / q^{2}\right)^{2}$.

To obtain the sound-sound correlations $D_{\sigma, \sigma^{\prime}}$, we require the corresponding elements of $H_{\sigma, \sigma^{\prime}}^{1}$. The only nonzero element is found to be

$$
H_{\sigma,-\sigma}^{1}=\frac{\sigma}{4 c_{s}^{2}}\left\{2 c_{s} q\left(\frac{\partial c_{s}}{\partial r_{x}}\right) \frac{\partial}{\partial q_{x}}-\hat{\mathbf{q}}_{x}\left[2 c_{s}^{3} \frac{\partial}{\partial r_{x}}+3 c_{s}^{2}\left(\frac{\partial c_{s}}{\partial r_{x}}\right)+A_{2} c_{s}\left(\frac{\partial A_{3}}{\partial r_{x}}\right)\right]\right\} .
$$

With this result the equation for the dominant correlation $D_{\sigma,-\sigma}$ is obtained after a straightforward but long algebraic calculation. The result is

$$
\left(-\Gamma_{s} q^{2}+\sigma q \frac{\partial c_{s}}{\partial r_{x}} \frac{\partial}{\partial q_{x}}-\sigma c_{s} \hat{\mathbf{q}}_{x} X^{T}-\sigma c_{s} \hat{\mathbf{q}}_{x} \frac{\partial}{\partial r_{x}}\right) \frac{2 \beta c_{s}^{2}}{\rho} D_{\sigma,-\sigma}=2 \beta c_{s}^{2}\left(\delta a_{\sigma}(\widehat{\mathbf{q}}) \delta a_{-\sigma}(-\hat{\mathbf{q}}) \mathbf{S}_{x T}\right) / \rho,
$$

where $\Gamma_{s}$ is given in Eq. $(\mathrm{C} 4)$ and

$$
\left(\delta a_{\sigma}(\hat{\mathbf{q}}) \delta a_{-\sigma}(-\hat{\mathbf{q}}) \mathbf{S}_{x T}\right)=\hat{\mathbf{q}}_{x} \frac{\sigma \rho}{2 \beta^{2} c_{s}} .
$$

The form of Eq. (6.22) is identical to the equation for $D_{\sigma,-\sigma}$ obtained in a one-component fluid ${ }^{5}$ except for minor changes. This similarity is expected due to the fact that the sound-sound correlation decouples from the dissipative-dissipative correlations. Besides the modification of $\Gamma_{s}$, the only difference is that for a binary mixture $c_{s}$ depends on $r$ through both $T$ and $c$. Now the gradient becomes

$$
\frac{\partial}{\partial r_{x}} c_{s}=\left[\left(\frac{\partial c_{s}}{\partial T}\right)_{c, p}-k_{T}\left(\frac{\partial c_{s}}{\partial c}\right)_{T, p}\right] X^{T} .
$$

However, the spatial variation of the sound velocity is not important since Brillouin scattering is best observed when the wave vector of the incident light is perpendicu- lar to the temperature gradient. For this scattering geometry the spatial variation of the sound velocity can be neglected. ${ }^{5}$ Consequently, only the modification in $\Gamma_{s}$ need be accounted for. Since these modifications do not affect functional form of the solution, the solution obtained for $D_{\sigma,-\sigma}$ in a one-component fluid is complete and we will not repeat the calculation here.

\section{TIME-DEPENDENT CORRELATIONS}

Once the gradient-dependent equal-time correlations have been found, the time-dependent correlations $M_{a b}(\mathbf{r}, \mathbf{q}, z)$ are easily calculated. The equations for the correlations in terms of the modes $M_{a b}(\mathbf{r}, \mathbf{q}, \mathbf{z})$, obtained from Eq. (5.2) by expanding in terms of the eigenvectors of $H^{0}$, are

$$
z M_{a b}-H_{a d}^{0} M_{d b}-H_{a d}^{1} M_{d b}=A_{a b}+D_{a b},
$$

where, as discussed in Appendix C, corrections to this 
equation are required for the sound-sound element $M_{\sigma, \sigma^{\prime}}$.

Repeating the analysis of Sec. VI, we again neglect the correlations coupling the propagating terms to the dissipative terms. The solutions obtained for the elements $M_{\eta, b}(\mathbf{r}, \mathbf{q}, z)$, where $b=\eta_{i}, H$, and $c$, are

$$
\begin{aligned}
& M_{\eta_{t} \eta_{l}}=A_{\eta_{t} \eta_{t}} /\left(z+v q^{2}\right), \\
& M_{\eta_{t} H}=D_{\eta_{t} H} /\left(z+v q^{2}\right),
\end{aligned}
$$

and

$$
M_{\eta_{l} c}=D_{\eta_{l} c} /\left(z+v q^{2}\right) .
$$

The correlations required for the light-scattering spectrum are $\boldsymbol{M}_{H H}(\mathbf{r}, \mathbf{q}, z), \quad \boldsymbol{M}_{c c}(\mathbf{r}, \mathbf{q}, z), \quad \boldsymbol{M}_{c H}(\mathbf{r}, \mathbf{q}, z)$, and $M_{H c}(\mathbf{r}, \mathbf{q}, z)$. The solutions obtained for these terms are

$$
\begin{aligned}
M_{H H}=A_{H H}\left(\dot{z}-H_{c c}^{0}\right) / \Delta+ & {\left[z+q^{2}\left(D_{T}+\mathcal{D}\right)\right] D_{H H} } \\
& \left.+\frac{q^{2}(\mathcal{D}-v)}{z+v q^{2}} H_{H \eta_{1}}^{1} D_{\eta_{1} H}\right) / \Delta,
\end{aligned}
$$

$$
\begin{aligned}
M_{c c}= & A_{c c}\left(z-H_{H H}^{0}\right) / \Delta \\
& +\left(\left[z+q^{2}\left(D_{T}+\mathcal{D}\right)\right] D_{c c}\right. \\
& \left.+\frac{q^{2}\left(D_{T}+\mathcal{D}-v\right)}{z+v q^{2}} H_{c \eta_{1}}^{1} D_{\eta_{1} c}\right) / \Delta,
\end{aligned}
$$

and

$$
\begin{aligned}
M_{H c}+M_{c H}= & \left(A_{H H} H_{c H}^{0}+A_{c c} H_{H c}^{0}\right) / \Delta \\
& +\left\lfloor\left[z+q^{2}\left(D_{T}+\mathcal{D}\right)\right]\left(D_{c H}+D_{H c}\right)\right. \\
& +\frac{q^{2}(D-v)}{z+v q^{2}} H_{H \eta_{1}}^{1} D_{\eta_{1} c} \\
& \left.+\frac{q^{2}\left(D_{T}+D-v-v\right)}{z+v q^{2}} H_{c \eta_{1}}^{1} D_{\eta_{1} H}\right] / \Delta,
\end{aligned}
$$

where

$$
\Delta=\left(z+z_{+}\right)\left(z+z_{-}\right) .
$$

The spatial, wave-vector, and $z$ dependence of the arguments has been neglected for the sake of simplicity. Note that in the absence of the temperature gradient the above expressions reduce to the usual equilibrium expressions. ${ }^{15}$

If we set $z=i \omega$ and substitute for $D_{a b}$ and $H_{a b}^{1}$ from Eqs. (6.9a) and (6.9b) and (6.18)-(6.20), we find, after some manipulation, that the expressions for the real part of the gradient-dependent correlations, denoted here by $\Delta M_{a b}$, simplify substantially. They are

$$
\begin{aligned}
\operatorname{Re}\left(\Delta M_{H H}\right)= & \frac{\rho\left(\alpha_{T} T X^{T} \hat{\mathbf{q}}_{\perp}^{(1)}\right)^{2} q^{2}}{\beta\left(\omega^{2}+z_{+}^{2}\right)\left(\omega^{2}+z_{-}^{2}\right)\left(\omega^{2}+v^{2} q^{4}\right)} \\
& \times\left(\omega^{2} v+v \mathcal{D}^{2} q^{4}\right),
\end{aligned}
$$

$$
\begin{aligned}
\operatorname{Re}\left(\Delta M_{c c}\right)= & \frac{\left(k_{T} X^{T} \hat{\mathbf{q}}_{\perp}^{(1)}\right)^{2} q^{2}}{\rho \beta\left(\omega^{2}+z_{+}^{2}\right)\left(\omega^{2}+z_{-}^{2}\right)\left(\omega^{2}+v^{2} q^{4}\right)} \\
& \times\left[\omega^{2} v+v\left(D_{T}+\mathcal{D}\right)^{2} q^{4}\right], \\
\operatorname{Re}\left(\Delta M_{c H}+\Delta M_{H c}\right)=- & \frac{k_{T} \alpha_{T} T\left(X^{T} \hat{\mathbf{q}}_{\perp}^{(1)}\right)^{2} q^{2}}{\beta\left(\omega^{2}+z_{+}^{2}\right)\left(\omega^{2}+z_{-}^{2}\right)\left(\omega^{2}+v^{2} q^{4}\right)} \\
& \times\left[2 \omega^{2} v+2 v \mathcal{D}\left(D_{T}+\mathcal{D}\right) q^{4}\right] .
\end{aligned}
$$

$\hat{\mathbf{q}}_{\perp}^{(1)}$ is defined in Eq. (6.10a).

The dynamic structure factor is obtained by combining the above results with original expressions (2.1) and (2.4). The spatial integrals appearing in the equation (2.4) for $S(\mathbf{k}, \omega)$ are done by assuming that the form factors $P(\mathbf{r})$ are sharply peaked functions at a point $\mathbf{R}$. When this assumption is made, the structure factor at the Rayleigh line is

$$
\begin{aligned}
S(\mathbf{R}, \mathbf{q}, w)=2\left[\left(\frac{\partial \epsilon}{\partial T}\right]_{p, c}^{2}\left(\rho \alpha_{T}\right)^{-2} \operatorname{Re}\left(\Delta M_{H H}\right)\right. \\
+\left(\frac{\partial \epsilon}{\partial c}\right]_{p, T}^{2} \operatorname{Re}\left(\Delta M_{c c}\right) \\
+\left(\frac{\partial \epsilon}{\partial T}\right)_{p, c}\left(\frac{\partial \epsilon}{\partial c}\right]_{p, T}\left(\rho \alpha_{T}\right)^{-1} \\
\\
\left.\times \operatorname{Re}\left(\Delta M_{c H}+\Delta M_{H c}\right)\right]
\end{aligned}
$$

where the thermodynamic relations (2.3) have been used to obtain the more conventional forms of the partial derivatives in $\epsilon$. If we note that the heat-heat correlation is simply related to temperature-fluctuation correlations by

$$
M_{H H}=\rho \alpha_{T}\langle\delta T(\omega, \mathbf{q}) \delta T(\omega,-\mathbf{q})\rangle,
$$

the above equation results are identical to the results we obtained previously ${ }^{12}$ using the theory of fluctuating hydrodynamics, ${ }^{19}$ except that any arbitrary direction for $q$ is considered here.

For the Brillouin lines the predicted spectrum for the binary fluid is trivially obtained from that of a onecomponent fluid. Since the operator $H_{\sigma,-\sigma}^{1}$ and the equal-time correlation $D_{\sigma,-\sigma}$ obtained here are identical in form to those of a one-component fluid, the correlation $M_{\sigma,-\sigma}$ is also identical. Again the only modification that need be made is that the expression for $\Gamma_{\varsigma}$ appropriate for a mixture be used and that spatial gradients of position-dependent variables be expanded to include both the concentration and temperature gradients.

\section{SUMMARY AND CONCLUSION}

Predictions of the effect of a large temperature gradient on fluctuation correlations and the dynamic structure factor in a binary liquid are made. Our predictions are based on the assumption that the macroscopic and microscopic time scales of the fluid are well separated. Conse- 
quently, mixtures close to their critical points are excluded. This assumption is shown to be equivalent to assuming that the Rayleigh and Brillouin peaks remain well separated, which permits us to consider the effect of the gradient at the Rayleigh and Brillouin lines separately.

The appropriate modes for a binary mixture are determined and the equal-time and time-dependent correlations of the fluctuations of the modes, subject to the gradient, are calculated. The resulting, lengthy expressions for the equal-time correlations are given by Eqs. (6.15)-(6.20) and by Eqs. (7.2)-(7.7) for the timedependent correlations. The prediction for the gradientdependent light-scattering spectrum is given by Eq. (7.12).

The effect of the temperature gradient at the Rayleigh line is similar to that obtained in a one-component fluid in that the change in the peak amplitude is proportional to the square of the gradients; terms of linear order leave the Rayleigh line unaffected. However, the dominant contribution to the spectrum is due to the coupling of concentration fluctuations where the coupling arises from two equally contributing sources, namely, the direct coupling of concentration modes and concentration coupling mediated by viscous modes. The results obtained here confirm our previous calculation for the Rayleigh line, ${ }^{12}$ which was made using the theory of fluctuating hydrodynamics.

Due to the contribution of concentration fluctuations, the effect in a mixture at the Rayleigh line is substantially larger than that of a one-component fluid. In Fig. 1 the dynamic structure factor for a mixture of benzene and carbon tetrachloride at a mole fraction 0.5 and a mean temperature of $300 \mathrm{~K}$ is shown. In the figure the equilibrium behavior, $\Delta T=0$, is compared with the behavior at a temperature gradient of $\Delta T=100 \mathrm{~K} / \mathrm{m}$ for two values

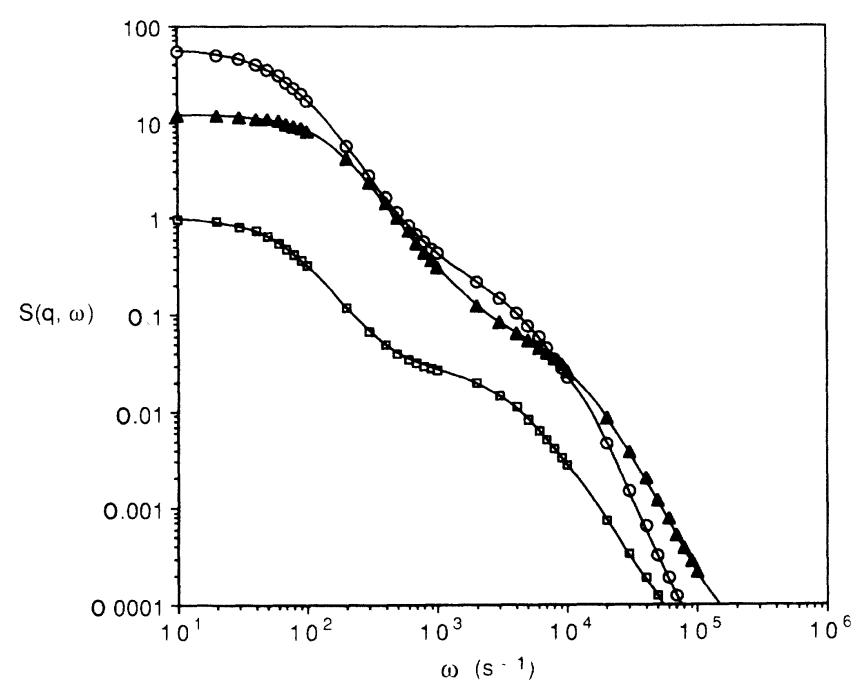

FIG. 1. The normalized structure factor for a 0.5-molefraction mixture of benzene and carbon tetrachloride at mean temperature $T=300 \mathrm{~K}$. The figure gives the behavior at equilibrium, $|\nabla T|=0$ and $q=2000 \mathrm{~cm}^{-1}$ (squares), and the nonequilibrium behavior for a temperature gradient $|\nabla T|=100$ $\mathrm{K} / \mathrm{cm}$ and for wave vectors $q=2000 \mathrm{~cm}^{-1}$ (circles) and $q=3000$ $\mathrm{cm}^{-1}$ (triangles). The equilibrium structure factor has been normalized to unity at $\omega=0$. of the wave number. The equilibrium data have been normalized to unity at $\omega=0$. The nonequilibrium enhancement for frequencies in the range $\omega=10-10^{3} \mathrm{~s}^{-1}$ is primarily due to concentration-concentration coupling, while the enhancement at higher frequencies, $\omega>10^{3} \mathrm{~s}^{-1}$ is due to heat-heat correlations. The structure factor at $q=2000 \mathrm{~cm}^{-1}$ (denoted by circles) decreases more rapidly than for $q=3000 \mathrm{~cm}^{-1}$ (triangles) because the characteristic frequencies $z_{+}$and $z_{-}$, given by $\mathrm{Eq}$. (6.17), scale as $q^{2}$. Note that the nonequilibrium spectrum is enhanced by a factor of the order of $10-10^{2}$ for the frequency range shown.

The predictions for the effect of the temperature gradient at the Brillouin line when compared to the effects at the Rayleigh line are negligible. Since the sound-sound correlations essentially decouple from the concentration and heat correlations even at large gradients, the Brillouin line is unaffected by the temperature gradient. Consequently, the predicted form for the Brillouin spectrum for the mixture is identical to the spectrum predicted in a one-component fluid.

\section{ACKNOWLEDGMENTS}

We would like to acknowledge helpful discussions with Professor Ted Kirkpatrick. This research was supported by the University of Southern Mississippi and partially supported by a grant from Research Corporation. Further support for this work was due to a NSF Grant No. DMR-8814439.

\section{APPENDIX A}

The equilibrium equal-time correlation functions required in this paper follow from Eq. (6.2). Further, we use the result ${ }^{17}$ that, in the long-wavelength limit $q \rightarrow 0$,

$$
\left(\delta a_{\alpha}(\mathbf{q}), \delta a_{\beta}(-\mathbf{q})\right)=\left(\frac{\partial \hat{a}_{\alpha}(\mathbf{r})}{\partial y_{\beta}(\mathbf{r})}\right)_{y_{v}(\mathbf{r})},
$$

where we define

$$
\widehat{a}_{\alpha}(\mathbf{r}) \equiv\left\langle a_{\alpha}(\mathbf{r})\right\rangle_{0} / V .
$$

With the above result the equal-time correlation functions follow from the expression for $F_{0}$ given in Eq. (3.13). The nonzero equilibrium correlations are

$$
\begin{aligned}
& A_{\eta_{t} b}=\left(\delta a_{\eta_{l}}(\hat{\mathbf{q}}), \delta a_{b}(-\hat{\mathbf{q}})\right)=-\frac{\rho}{\beta c_{s}^{2}} \delta_{\eta_{l} b}, \\
& A_{H b}=\left(\delta a_{H}(\hat{\mathbf{q}}), \delta a_{b}(-\hat{\mathbf{q}})\right)=\frac{\rho \alpha_{T}^{2} T}{\beta C_{p, c}} \delta_{H b}, \\
& A_{c b}=\left(\delta a_{c}(\hat{\mathbf{q}}), \delta a_{b}(-\hat{\mathbf{q}})\right)=\frac{1}{\beta \rho}(\partial c / \partial \mu)_{p, T} \delta_{c b}, \\
& A_{\sigma, \sigma^{\prime}}=\left(\delta a_{\sigma}(\hat{\mathbf{q}}), \delta a_{\sigma^{\prime}}(-\hat{\mathbf{q}})\right)=\frac{\rho}{2 \beta c_{s}^{2}} \delta_{\sigma^{\prime},-\sigma} .
\end{aligned}
$$

The projected flux terms are calculated directly from the definitions for the fluxes, Eqs. (6.4) and (6.5), and the definitions of the conserved densities. When the assumption is made that the averaged flow $\mathbf{u}=0$, the fluxes associated with the density components $\rho_{l}$ are found to be 


$$
\mathbf{I}_{\rho_{J}, l T}=\mathbf{g}_{j, I T}-\frac{\rho_{j, T}}{\rho_{T}} \mathbf{g}_{i T}, \quad j=1,2 .
$$

Here and throughout the rest of this appendix the subscript $i$ will denote the $i$ th component of a vector. The subscript $T$ denotes the total quantity, defined by Eq. (3.13a). For example,

$$
\begin{aligned}
& \mathbf{g}_{i T}=\mathbf{g}_{i}(\mathbf{k}=0, t)=\sum_{j=1}^{N} \mathbf{p}_{\imath}, \\
& \mathbf{g}_{j, \imath T}=\mathbf{g}_{j, \imath}(\mathbf{k}=0, t)=\sum_{i=1}^{N_{j}} \mathbf{p}_{i} .
\end{aligned}
$$

Note that

$$
\mathbf{I}_{\rho, I T}=\mathbf{I}_{\rho_{1}, l T}+\mathbf{I}_{\rho_{2}, i T}=\mathbf{0} .
$$

The energy density flux is given by

$$
\mathbf{I}_{\varepsilon, i T}=\sum_{j=1}^{N} e_{j} \mathbf{v}_{i, j}+\sum_{j \neq k}^{N}\left(\mathbf{v}_{i, j}+\mathbf{v}_{i, k}\right) \mathbf{r}_{j k} \cdot \frac{\partial}{\partial \mathbf{r}_{j k}} \phi-h_{T} \mathbf{g}_{i T},
$$

where $h_{T}$ is the total enthalpy per unit mass, namely, $h_{T}=\hat{h}_{T} / \rho_{T}$, and the enthalpy per unit volume is given by $\hat{h}_{T}=\varepsilon_{T}+p$. The velocity of the $i$ th particle $\mathbf{v}_{j}=\mathbf{p}_{j} / m_{j}$. The term required in Eq. (6.3) is $I_{\alpha, i T} \partial y_{\alpha}(\mathbf{r}) / \partial \mathbf{r}_{l}$. Using Eq. (4.2) and standard thermodynamic identities for binary mixtures, we find

$$
I_{v, l T} \frac{\partial}{\partial \mathbf{r}_{\imath}} y_{v}(\mathbf{r})=\beta X^{T} \mathbf{S}_{x}
$$

where the subscript $x$ denotes the $x$ component and

$$
\mathbf{S}_{x}=\mathbf{I}_{x}^{\lambda}+k_{T}\left(\frac{\partial \mu}{\partial c}\right)_{p, T} \mathbf{I}_{x}^{k}
$$

with

$$
\mathbf{I}_{x}^{\lambda}=\mathbf{I}_{\varepsilon, x T}-h_{1} \mathbf{g}_{1, x T}-h_{2} \mathbf{g}_{2, x T}
$$

and

$$
\mathbf{I}_{x}^{k}=\mathbf{g}_{1, x T}-c \mathbf{g}_{x T} .
$$

Here $h_{j}$ is the enthalpy per unit mass of mixture component $j$. Using the above results we find for the modemode-flux correlations that

$$
\begin{aligned}
\left(\delta a_{\eta_{l}}(\hat{\mathbf{q}}) \delta a_{\eta_{j}}(-\hat{\mathbf{q}}) \mathbf{S}_{x T}\right) & =\left(\delta a_{H}(\hat{\mathbf{q}}) \delta a_{H}(-\hat{\mathbf{q}}) \mathbf{S}_{x T}\right) \\
& =\left(\delta a_{c}(\hat{\mathbf{q}}) \delta a_{c}(-\hat{\mathbf{q}}) \mathbf{S}_{x T}\right)=0, \\
\left(\delta a_{\eta_{l}}(\hat{\mathbf{q}}) \delta a_{H}(-\hat{\mathbf{q}}) \mathbf{S}_{x T}\right) & =-\hat{\mathbf{q}}_{\perp x}^{(l)} \rho \alpha_{T} T / \beta^{2} c_{s}, \\
\left(\delta a_{\eta_{l}}(\hat{\mathbf{q}}) \delta a_{c}(-\hat{\mathbf{q}}) \mathbf{S}_{x T}\right) & =\hat{\mathbf{q}}_{\perp x}^{(i)} k_{T} / \beta^{2} c_{s}, \\
\left(\delta a_{\sigma}(\hat{\mathbf{q}}) \delta a_{\sigma^{\prime}}(-\hat{\mathbf{q}}) \mathbf{S}_{x T}\right) & =\delta_{\sigma^{\prime},-\sigma} \sigma \rho \hat{\mathbf{q}}_{x} / 2 \beta^{2} c_{s} .
\end{aligned}
$$

\section{APPENDIX B: MODES OF THE EQUILIBRIUM MATRIX $H_{\alpha \beta}^{0}$}

In matrix form the fluctuation variables are represented by $\delta a^{R}=\left(\begin{array}{c}\delta \rho \\ \delta c \\ \delta g_{x} \\ \delta g_{y} \\ \delta g_{z} \\ \delta \varepsilon\end{array}\right), \quad \delta a^{L}=\left(\delta \rho, \delta c, \delta g_{x}, \delta g_{y}, \delta g_{z}, \delta \varepsilon\right)$

In this representation the eigenvalues $\lambda_{\alpha}$ and the left and right eigenvectors of $H_{\alpha \beta}^{0}$ are defined by

$$
H_{\alpha \beta}^{0} \theta_{\beta}^{R}=-\lambda_{\alpha} \theta_{\alpha}^{R}, \quad \theta_{\beta}^{L} H_{\beta \alpha}^{0}=-\lambda_{\alpha} \theta_{\alpha}^{L} .
$$

The orthogonality condition for the eigenvectors is $\theta_{\alpha}^{L} \cdot \theta_{\beta}^{R}=\delta_{\alpha \beta}$. The eigenvalues and eigenvectors are calculated to be

(a) Sound.

$$
H_{s, s}^{0} \equiv-\lambda_{s}=-\sigma c_{s} q-\Gamma_{s} q^{2} / 2,
$$

where $\Gamma_{s}$ is the sound damping constant given by

$$
\begin{aligned}
\Gamma_{s}= & {\left[\frac{4}{3} v+\frac{\xi}{\rho}\right]+D_{T}(\gamma-1) } \\
& +D \rho^{2} c_{s}^{2}\left(\frac{\partial \mu}{\partial c}\right)_{p, T}\left(\frac{\alpha_{T} k_{T}}{\rho C_{p, c}}+\frac{k_{p}}{p}\right)^{2}
\end{aligned}
$$

and

$$
\begin{aligned}
& \gamma=C_{V, c} / C_{p, c}, \\
& \theta_{\sigma}^{R}(\hat{\mathbf{q}})=\left(\begin{array}{c}
1 \\
0 \\
\sigma c_{s} \hat{q}_{x} \\
\sigma c_{s} \hat{q}_{y} \\
\sigma c_{s} \hat{q}_{z} \\
A_{3}
\end{array}\right), \\
& \theta_{\sigma}^{L}(\hat{q})=\frac{1}{2 c_{s}^{2}}\left(A_{1}, A_{1}^{\prime}, \sigma c_{s} \hat{q}_{x}, \sigma c_{s} \hat{q}_{y}, \sigma c_{s} \hat{q}_{z}, A_{2}\right),
\end{aligned}
$$

where $\sigma= \pm 1$. The sound mode is given by

$$
\delta a_{\sigma}(\mathbf{q}) \equiv \theta_{\sigma}^{L} \cdot \delta a^{R}=\frac{1}{2 c_{s}^{2}}\left(\delta p+\sigma c_{s} \hat{q} \cdot \mathbf{g}\right) .
$$

(b) Viscous.

$$
\begin{aligned}
& H_{\eta_{l}, \eta_{j}}^{0} \equiv-\lambda_{\eta_{t}} \delta_{l, j}=-v q^{2} \delta_{l, j}, \\
& \theta_{\eta_{l}}^{R}(\hat{\mathbf{q}})=c_{s}\left(\begin{array}{c}
0 \\
0 \\
\hat{q}_{\perp x}^{(i)} \\
\hat{q}^{(i)} \\
1 y \\
\hat{q}_{\perp z}^{(i)} \\
0
\end{array}\right), \\
& \theta_{\eta_{l}}^{L}(\hat{\mathbf{q}})=\frac{1}{c_{s}}\left(0,0, \hat{q}_{\perp x}^{(l)}, \hat{q}_{\perp y}^{(i)}, \hat{q}_{\perp z}^{(i)}, 0\right),
\end{aligned}
$$

where $\hat{\mathbf{q}}_{\perp}^{(i)}$ is defined by Eqs. (6.10a) and (6.10b). The 
viscous mode is given by

$$
\delta a_{\eta_{l}}(\mathbf{q}) \equiv \theta_{\eta_{t}}^{L} \cdot \delta a^{R}=\frac{1}{c} \hat{\mathbf{q}}_{\perp}^{(1)} \cdot \mathbf{g}
$$

(c) Heat.

$$
\begin{aligned}
& \theta_{H}^{L}(\hat{\mathbf{q}})=\frac{A_{2}}{c_{s}^{2}}\left[A_{3}, A_{3}^{\prime}+\rho T\left(\frac{\partial s}{\partial c}\right]_{p, T}, 0,0,0,-1\right), \\
& \theta_{H}^{R}=\left(\begin{array}{c}
1 \\
0 \\
0 \\
0 \\
0 \\
-A_{1} / A_{2}
\end{array}\right),
\end{aligned}
$$

and the heat mode is given by

$$
\delta a_{H}=\theta_{H}^{L} \cdot \delta a^{R}=-\frac{\rho T A_{2}}{c_{s}^{2}} \delta s^{\prime},
$$

where $\delta s^{\prime}$ is the modified entropy variable defined by Eq. (2.2).

(d) Concentration.

$$
\theta_{c}^{R}=\left(\begin{array}{c}
-c_{x}^{2} / c_{s}^{2} \\
1 \\
0 \\
0 \\
0 \\
\frac{A_{1} c_{x}^{2}}{A_{2} c_{s}^{2}}-\frac{A_{1}^{\prime}}{A_{2}}
\end{array}\right), \quad \theta_{c}^{L}=(0,1,0,0,0,0),
$$

where

$$
c_{x}^{2}=\left(\frac{\partial p}{\partial c}\right)_{s, \rho}+\rho T A_{2}\left(\frac{\partial s}{\partial c}\right)_{p, T}
$$

and the concentration mode is

$$
\delta a_{c}=\delta c
$$

$H^{0}$ is not diagonalized by the heat and concentration eigenvectors, but has elements

$$
\begin{aligned}
& H_{H H}^{0}(q)=-q^{2}\left[D_{T}+\frac{D k_{T}^{2}}{T C_{p, c}}\left(\frac{\partial \mu}{\partial c}\right]_{p, T}\right], \\
& H_{H c}^{0}(q)=q^{2} \frac{\rho \alpha_{T} D k_{T}}{C_{p, c}}\left(\frac{\partial \mu}{\partial c}\right)_{p, T}, \\
& H_{c H}^{0}(q)=q^{2} \frac{D k_{T}}{\rho \alpha_{T} T},
\end{aligned}
$$

and

$$
H_{c c}^{0}(q)=-q^{2} D
$$

\section{APPENDIX C}

In this appendix the relation between Eqs. (5.2) and (6.8a) is examined. We noted in the text that Eq. (6.8a), which is obtained by expanding (5.2) in terms of the eigenvectors, is not entirely correct. The required corrections are due to differential operators in $H^{1}$ which act on the spatially dependent variables appearing in the eigenvectors when the eigenvector expansion is made. To simplify the algebra it is convenient to rewrite Eq. (6.3) in matrix notation, as follows:

$$
H \cdot D+D \cdot \widetilde{H}=X,
$$

where

$$
X=\left(\delta a^{L}(\hat{\mathbf{q}}) \cdot \delta \widetilde{a}^{L}(\hat{\mathbf{q}}) I_{v, I T}\right) \frac{\partial}{\partial \mathbf{r}_{i}} y_{v}(\mathbf{r})
$$

and $\delta a^{L}(\widehat{\mathbf{q}})$ is defined in Eq. (B1). Here

$$
\delta \widetilde{a}^{L}(\hat{\mathbf{q}})=\delta a^{L}(-\hat{\mathbf{q}})^{t}
$$

and $\widetilde{H}(\mathbf{q})=H(-\mathbf{q})^{t}$. The superscript $t$ denotes the transposed matrix and the dot product denotes matrix multiplication. Further, the differential operators in $\widetilde{H}$ are defined to act only on the terms preceding it. Due to the orthogonality of the eigenvectors, we can write that

$$
\theta_{a}^{R} \cdot \theta_{a}^{L}=\widetilde{\theta}_{a}^{L} \cdot \tilde{\theta}_{a}^{R}=I,
$$

where the repeated index notation denotes summation. I is a $6 \times 6$ unit matrix and $\widetilde{\theta}_{a}(\hat{\mathbf{q}})=\theta_{a}(-\hat{\mathbf{q}})^{t}$. Expanding Eq. $(\mathrm{C} 1)$ in terms of the modes, we find

$$
\begin{aligned}
X_{a b}=\theta_{a}^{L} \cdot X \cdot \theta_{b}^{R}= & \left(\theta_{a}^{L} \cdot H \cdot \theta_{d}^{R} \cdot \theta_{d}^{L} \cdot D\right) \cdot \widetilde{\theta}_{b}^{L} \\
& +\theta_{a}^{L} \cdot\left(D \cdot \widetilde{\theta}_{d}^{L} \cdot \widetilde{\theta}_{d}^{R} \cdot \widetilde{H}^{\prime} \cdot \widetilde{\theta}_{b}^{L}\right),
\end{aligned}
$$

where the differential operators in $H$ act to the right on the terms within the parentheses, and differential operators in $\tilde{H}$ act to the left on the terms within the parentheses. When the eigenvectors outside the parentheses are included, the equation becomes

$$
\begin{aligned}
X_{a b}= & H_{a d} D_{d c}+D_{a d} \widetilde{H}_{d c}-\left(\theta_{d}^{L} \cdot D\right)_{k} H_{a d}^{1} \theta_{b, k}^{L}+D_{d b} H_{a d}^{1} \\
& -\theta_{a, k}^{L} \widetilde{H}_{d b}^{1}\left(D \cdot \theta_{c}^{L}\right)_{k}+\widetilde{H}_{c b}^{1} D_{a c},
\end{aligned}
$$

which differs from Eq. (6.8a) by the last four correction terms. It is important to emphasize the definitions of the elements of $H$,

$$
\begin{aligned}
& H_{a b} \equiv \theta_{a}^{L} \cdot H \cdot \theta_{b}^{R}, \\
& \widetilde{H}_{a b} \equiv \widetilde{\theta}_{a}^{R} \cdot \widetilde{H} \cdot \widetilde{\theta}_{b}^{L}=\theta_{b}^{L}(-\widehat{\mathbf{q}}) \cdot H(-\mathbf{q}) \cdot \theta_{b}^{R}(-\hat{\mathbf{q}}),
\end{aligned}
$$

and

$$
D_{a b}=\theta_{a}^{L} \cdot D \cdot \tilde{\theta}_{b}^{R} .
$$

No corrections are needed for those elements in $H^{1}$ that do not contain differential operators. For example, no 
correction will apply to the element $H_{c g_{i}}^{1}$.

When $H_{a b}^{1}$ is calculated for the case where $a$ and $b$ denote dissipative modes, it is found that $H_{a b}^{1}$ no longer contains differential operators. Consequently, the last four correction terms in (C5) cancel and we reobtain Eq. (6.8a). However, if $a$ and $b$ denote sound modes, $H_{\sigma,-\sigma}^{1}$ which is given in Eq. (6.21), still contains differential operators and the correction terms become important.
*Present address: Shell Development Company, Bellaire Research Center, P.O. Box 481, Houston, TX 77001.

${ }^{1}$ A.-M. S. Tremblay, in Recent Developments in Nonequilibrium Thermodynamics, Vol. 199 of Lecture Notes in Physics, edited by J. Casas-Vazquez, D. Jou, and G. Lebron (SpringerVerlag, Berlin, 1984), p. 267.

${ }^{2}$ T. R. Kirkpatrick, E. G. D. Cohen, and J. R. Dorfman, Phys. Rev. Lett. 44, 472 (1980).

${ }^{3}$ T. R. Kirkpatrick, E. G. D. Cohen, and J. R. Dorfman, Phys. Rev. A 26, 950 (1982).

${ }^{4}$ T. R. Kirkpatrick, E. G. D. Cohen, and J. R. Dorfman, Phys. Rev. A 26, 972 (1982).

${ }^{5}$ T. R. Kirkpatrick, E. G. D. Cohen, and J. R. Dorfman, Phys. Rev. A 26, 995 (1982).

${ }^{6}$ D. Ronis and I. Procaccia, Phys. Rev. A 26, 1812 (1982).

${ }^{7}$ R. Schmitz and E. G. D. Cohen, J. Stat. Phys. 39, 285 (1985); 40, 431 (1985); 46, 319 (1987).

${ }^{8}$ B. M. Law, R. W. Gammon, and J. V. Sengers, Phys. Rev. Lett. 60, 1554 (1988).

${ }^{9}$ B. M. Law, P. N. Segre, R. W. Gammon, and J. V. Sengers, Phys. Rev. A 41, 816 (1990).
${ }^{10}$ D. Beysens, Y. Garrabos, and G. Zalcer, Phys. Rev. Lett. 45, 403 (1980).

${ }^{11}$ H. Kiefte, M. J. Clouter, and R. Penney, Phys. Rev. B 30, 4017 (1984)

${ }^{12}$ B. L. Law and J. C. Nieuwoudt, Phys. Rev. A 40, 3880 (1989).

${ }^{13}$ See, for example, B. J. Berne and R. Pecora, Dynamic Light Scattering with Applications to Chemistry, Biology and Physics (Wiley, New York, 1976).

${ }^{14}$ C. Cohen, J. W. H. Sutherland, and J. M. Deutch, Phys. Chem. Liquids 2, 213 (1971).

${ }^{15}$ R. D. Mountain and J. M. Deutch, J. Chem. Phys. 50, 1103 (1969).

${ }^{16}$ See, for example, L. D. Landau and E. M. Lifshitz, Fluid Mechanics (Pergamon, Oxford, 1984).

${ }^{17}$ M. H. Ernst, E. H. Hauge, and J. M. J. van Leeuwen, J. Stat. Phys. 15, 7 (1976).

${ }^{18} \mathrm{~A}$ more detailed exposition of the decoupling of the dissipative and sound mode elements in the equations for $D$ and $M$ is given in Ref. 3.

${ }^{19}$ The results of Ref. 12 differ numerically from Eq. (7.12) by an overall factor of $8 \pi^{2}$ due to the definition of $S(\mathbf{k}, \omega)$. 